\title{
A randomized controlled trial comparing the efficacy of tigecycline versus meropenem in the treatment of postoperative complicated intra-abdominal infections
}

\author{
Hai-Jun Wang^, Xue-Zhong Xing, Shi-Ning Qu, Chu-Lin Huang, Hao Zhang, Hao Wang, \\ Quan-Hui Yang, Zhen-Nan Yuan
}

Intensive Care Unit, National Cancer Center/National Clinical Research Center for Cancer/Cancer Hospital, Chinese Academy of Medical Sciences and Peking Union Medical College, Beijing, China

Contributions: (I) Conception and design: HJ Wang, XZ Xing; (II) Administrative support: XZ Xing, HJ Wang; (III) Provision of study materials or patients: HJ Wang; (IV) Collection and assembly of data: HJ Wang; (V) Data analysis and interpretation: HJ Wang; (VI) Manuscript writing: All authors; (VII) Final approval of manuscript: All authors.

Correspondence to: Xue-Zhong Xing. Intensive Care Unit, National Cancer Center/National Clinical Research Center for Cancer/Cancer Hospital, Chinese Academy of Medical Sciences and Peking Union Medical College, Beijing, China. Email: xingxzh2000@aliyun.com.

\begin{abstract}
Background: The efficacy and safety of tigecycline in the treatment of complicated intra-abdominal infections (cIAIs) is potentially controversial. Here we conducted the non-inferiority study to assess the efficacy and safety of tigecycline versus meropenem in the treatment of postoperative cIAIs.
\end{abstract}

Methods: Data of abdominal tumor surgery patients with postoperative cIAIs admitted to intensive care unit (ICU) between October 2017 and December 2019 were collected. A prospective, randomized controlled trial was conducted in which 56 eligible patients with cIAIs randomly received intravenous tigecycline or meropenem for 3 to 14 days. Patients and clinicians were not blinded to the group allocation.

Results: The total of 56 patients were enrolled, which were divided into 2 groups, one group included 30 patients receiving meropenem and another group included 26 receiving tigecycline therapy. The 2 groups were similar at demographic and baseline clinical characteristics. Microorganisms were isolated from 46 of 56 patients $(82.14 \%)$, with a total of 107 pathogens were cultured in two groups. The two groups had similar distribution of infecting microorganisms. The primary end point was the clinical response at the end-oftherapy (EOT) visit and upon discharge visit and comprehensive efficacy. The clinical success rates were $83.33 \%, 76.67 \%$ for meropenem versus $76.92 \%, 88.46 \%$ for tigecycline at the EOT visit and upon discharge visit $(\mathrm{P}>0.05)$, respectively. Comprehensive efficacy did not significantly differ between two groups either. There were no significant differences in 30-day and 60-day all-cause mortality between two groups $(\mathrm{P}>0.05)$. The univariable analysis identified that serum albumin at admission ICU, colorectal cancer on oncology type, postoperative abdominal bleeding were the risk factors for 60-day all-cause mortality. The multivariable analysis showed that postoperative abdominal bleeding were independent predictors of 60-day all-cause mortality. Gastrointestinal disorders and antibacterials-induced Fungal Infection were the most frequently reported adverse events (AEs). The incidence of AEs was similar between meropenem and tigecycline groups $(\mathrm{P}>0.05)$.

Conclusions: Taken together, the study demonstrated that tigecycline is as effective and safe as meropenem for postoperative cIAIs in abdominal tumors patients. Tigecycline is non-inferior to meropenem.

Keywords: Tigecycline; meropenem; cancer complicated intra-abdominal infections effect (cancer cIAIs effect)

Submitted Apr 09, 2020. Accepted for publication Sep 05, 2020.

doi: 10.21037/apm-20-907

View this article at: http://dx.doi.org/10.21037/apm-20-907

^ ORCID: 0000-0002-7260-006X. 


\section{Introduction}

Complicated intra-abdominal infections (cIAIs) were defined as the infectious extended to peritoneal cavity and caused either localised or diffuse peritonitis (1). The management of cIAIs remains challenging because of their polymicrobial nature and heterogenicity (2). Previous studies have indicated that the mortality of cIAIs was up to $10.5 \%$ (3). Hence, timely and effective treatment of cIAIs is crucial, including early recognition and fluid resuscitation, adequate source control, and appropriate antimicrobial therapy (4). Notably, the empiric antibiotics plays a significant role for patients with cIAIs, particularly those with hospital-acquired intra-abdominal infections which have high risk factors for multidrug-resistant (MDR) organisms infection (5). Meropenem, is an important option for the empirical treatment of cIAIs that exhibits a wide spectrum of activity against a variety of organisms, including polymicrobial, anaerobic bacteria and resistant flora that cause cIAIs. Moreover published treatment guidelines recommend meropenem as monotherapy or combination regimens for postoperative cIAIs (6), which is why we chose it as a comparator. However, empirical antibiotic therapy has been complicated by the growing emergence of drug-resistant organisms, particularly extended-spectrum $\beta$-lactamase-producing enterobacteriaceae, and by the overuse of carbapenemresistant bacteria, especially carbapenem-resistant klebsiella pneumoniae. Particularly, carbapenem resistance is becoming increasingly prevalent. Therefore, it is necessary to limit the use of carbapenem in order to maintain its antibacterial activity. Then we need to find an alternative antimicrobial agent to cover these frequently isolated pathogens and difficult-to-treat isolates causing cIAIs effectively.

Tigecycline, a broad spectrum of antibacterial activity, remains a viable treatment option for cIAIs due to its favorable in vitro activity against Gram-positive and Gram-negative bacteria, and anaerobes, including MDR bacteria (7). In our previous study, we found that tigecycline was superior to meropenem with reduced hospital mortality in abdominal tumours patients with cIAIs. However, the retrospective nature of the study made it low level evidence. We hypothesized that there is no difference in outcomes and safety between tigecycline and meropenem therapy in patients with cIAIs. The present study was designed to prospectively assess the efficacy and safety of tigecycline versus meropenem in the treatment of postoperative abdominal tumors patients with cIAIs. We present the following study in accordance with the CONSORT (Consolidated Standards of Reporting Trails) reporting checklist (8). Available at http://dx.doi.org/10.21037/apm20-907.

\section{Methods}

\section{Ethical approval}

The study was conducted accordance with the Declaration of Helsinki (as revised in 2013) and was approved by the Independent Ethics Committee of National Cancer Center/ Cancer Hospital, Chinese Academy of Medical Sciences and Peking Union Medical College (No. NCC2018W-001). Informed written consent was obtained from each patient or his or her legal representative prior to their enrollment in this study.

\section{Study population}

This was a prospective, single-center open-labeled, randomized controlled trial conducted in a tertiary cancer hospital in china. Clinical data were collected from 76 patients diagnosed as postoperative cIAIs in abdominal tumor patients between October 2017 and December 2019 in intensive care unit (ICU). Twenty patients were excluded because of non-compliance with entry criteria, finally, 56 patients were recruited to the trial.

\section{Study procedures}

We used the simple randomization method. Patients were randomly assigned (1:1) using random number table method and divided into meropenem group (30 cases) and tigecycline group (26 cases). Patient's allocation was determined by a trial researcher (WHJ). Meropenem was administered with IV dose of $1,000 \mathrm{mg}$ every 8 hours or dose-adjusted based on weight and creatinine clearance, tigecycline was administered with an initial IV dose of $100 \mathrm{mg}$ followed by $50 \mathrm{mg}$ twice a day, approximately every 12 hours. Consider use of IV vancomycin added if there is suspected or confirmed infection with Gramnegative bacteria such as Enterococcus spp., and methicillinresistant Staphylococcus aureus (MRSA) etc. in meropenem group. Vancomycin was administered with IV dose of $1,000 \mathrm{mg}$ every 12 hours or dose-adjusted based on weight and creatinine clearance. If culture results isolated from 
tigecycline group organisms identified as Pseudomonas aeruginosa and Proteus spp, patients in the tigecycline group were treated with tigecycline plus cefoperazonesulbactam (cefoperazone 2,000 $\mathrm{mg}$ and sulbactam 1,000 mg intravenously every $12 \mathrm{~h}$ daily). Co-infection with fungus in two groups was administered antifungal agents.

\section{Inclusion criteria}

All consecutive patients with cIAIs were screened for inclusion in the study. The inclusion criteria included: (I) both men and women patients were $\geq 18$ years old; (II) clinical diagnosis of postoperative cIAIs; (III) the diagnosis of cIAIs is based primarily on clinical assessment (9). CIAIs must meet at least 1 of the following criteria: (I) patient has organisms cultured from purulent material from intraabdominal space obtained during a surgical operation or needle aspiration; (II) patient has abscess or other evidence of intraabdominal infection seen during a surgical operation or histopathologic examination; (III) patients have at least 2 of the following signs or symptoms with no other recognized cause: fever $\left(>38^{\circ} \mathrm{C}\right)$, nausea, vomiting, abdominal pain, or jaundice and at least 1 of the following: (A) organisms cultured from drainage from surgically placed drain (e.g., closed suction drainage system, open drain, T-Tube drain); (B) organisms seen on Gram's stain of drainage or tissue obtained during surgical operation or needle aspiration; (C) organisms cultured from blood and radiographic evidence of infection [e.g., abnormal findings on ultrasound, CT scan, MRI, or radiolabel scans (gallium, technetium, etc.) or on abdominal X-ray] (10).

\section{Exclusion criteria}

The following exclusions applied: (I) patients who are known to have a suspected allergy or serious adverse reaction to meropenem and tigecycline or a similar drug; (II) had an anticipated length of antibiotic therapy <3 days; (III) an ICU stay of $<48$ hours; (IV) had intolerable adverse events (AEs) or serious events occurred (SAEs) during treatment; (V) had other site infections requiring other antibiotic therapy on ICU admission.

\section{Data collection and evaluations}

All clinical data were recorded into a case report form (CRF) by the attending physicians. Data were collected including gender, age, underlying disease, cancer type (gastric cancer, pancreatic cancer, hepatobiliary neoplasm, colorectal cancer), type of cIAIs (anastomotic fistula, intra-abdominal abscess, diffuse peritonitis), APACHE II scores and SAPS3 scores and SOFA scores on ICU admission, multiple organ support in ICU [vasoactive agents, mechanical ventilation, continuous veno-venous hemofiltration $(\mathrm{CVVH})]$, the presence of septic shock and acute kidney injury (AKI) bloodstream infections, readmission to ICU unplanned reoperation, de-escalation, source control, initial appropriate antibiotics, type of micro-organisms, MDR organisms, hospital length of stay (LOS), ICU-LOS, antibiotics duration, postoperative abdominal bleeding.

Bloodstream infections was defined as at least 1 positive blood culture for a recognized pathogen and clinical symptoms consistent with bacteraemia (11). Readmission to ICU (12) was defined as any return to ICU within 2 calendar days after ICU discharge, during the index hospitalization. Initial appropriate antibiotics was defined as an initially prescribed antimicrobial regimen, tigecycline and meropenem, shows in vitro activity against all identified isolates prior to the availability of susceptibility information and administration within $24 \mathrm{~h}$ of cultures collection (13). MDR organism was defined as a bacterial isolate with in vitro resistance to at least three or more antimicrobial categories (14). Postoperative abdominal bleeding was defined as a decrease in hemoglobin of more than $3 \mathrm{~g} / \mathrm{dL}$ compared to preoperative values (15).

\section{Microbiological collection and definition}

All isolates were collected and cultured from specimens obtained from tissue, intraperitoneal fluid, deep wound, fluid from paracentesis or percutaneous aspiration of abscesses, blood on intraoperative and postoperative duration. All specimens were submitted on guidelines procedure during ICU period and were subjected to standard microbiological procedures in laboratory at our hospital to identify Gram-negative, Gram-positive, and anaerobes bacteria and fungi (16).

\section{Clinical assessments and definitions}

The primary outcome was clinical success rate and comprehensive efficacy by the investigator at end-oftherapy visit (EOT) (within 24 hours of the last dose) and upon discharge visit.

Clinical outcome was classified at the follow-up visits as success or failure according to Complicated Intra- 
Abdominal Infections: Developing Drugs for Treatment Guidance for Industry for clinical trials drawn up by the Food and Drug Administration (FDA) (17). Patients were considered "clinical success" if complete resolution or significant improvement of the initial clinical signs caused by the infection without additional antimicrobial therapy or intervention.

Patients considered to present "clinical failure" were those with signs of persistent or worsening infection, additional intervention and/or antibiotic therapy or who died as a result of cIAIs at any time. Comprehensive efficacy assessment was defined as the composite clinical outcome was evaluated only for the patients with positive microorganisms culture at EOT and upon discharge visit and categorized as success, failure.

The secondary outcomes evaluated hospital LOS, ICU LOS, 30-day all-cause mortality, 60-day all-cause mortality and incidence rates of AEs in two groups.

\section{Sample size and statistical analysis}

This sample size is estimated by the Power Analysis and Sample Size for Windows software (PASS 11.0, NCSS, Kaysville, Utah, USA). This study was based on the conception that tigecycline would be non-inferior to meropenem in relation to the primary efficacy outcome. On the basis of previous retrospective study from our hospital (18), we assumed that an estimated clinical success rate of 80 percent with the comparator, a prespecified non-inferiority margin not exceed 20 percentage points, a statistical power of $80 \%$, and a significance level of $0.1 \%$ (two sided). The resulting sample size was 36 in each group. A total of 76 patients were enrolled in this study. All analyses were performed using SPSS software program version 19.0 for window (SPSS Inc., USA). Categorical variables were expressed as frequency, percentages, or rate, and were compared using chi-squared test or Fisher's exact test. Student's $t$-test was used to compare the continuous variables. To control the effects of confounding variables and identify the independent risk factors associated with 60-day death. A stepwise conditional logistic regression analysis was performed. Variables with significant factors were entered into a multivariate logistic regression model to identify factors independently influencing unfavorable clinical outcomes. All variables with a $\mathrm{P}$ value $<0.05$ in univariate analysis were included in the multivariate logistic model to identify factors independently influencing unfavorable clinical outcomes. OR and $95 \%$ CI were calculated. $\mathrm{P}$ values $<0.05$ were considered statistically significant, and all tests were two-tailed.

\section{Results}

\section{Patient characteristics}

A total of 76 patients were originally recruited to the trial, of these, 20 patients were excluded because of the presence of length of antibiotic $<3$ days or ICU LOS $<48$ hours (Figure 1 CONSORT flow diagram). A total of 56 patients were finally enrolled, in meropenem group, there were 22 (73.33\%) male patients and 8 (26.67\%) female patients. the mean $( \pm \mathrm{SE})$ age of the patients was $63.37 \pm 10.95$ years; in tigecycline group, there were $21(80.77 \%)$ male patients and $5(19.23 \%)$ female patients and the mean $( \pm$ SE) age of the patients was $66.35 \pm 10.43$ years. The demographic and clinical characteristics were similar in the two groups (Table 1).

\section{Clinical outcome}

\section{The primary outcomes}

Clinical success rates for meropenem and tigecycline at EOT visit were $83.33 \%$ and $76.92 \%$, respectively, and there was no significant difference $(\mathrm{P}>0.05)$. Clinical success rates at upon discharge visit were $76.67 \%$ for the meropenem group, compared with $88.64 \%$ in tigecycline group $(\mathrm{P}>0.05)$. Clinical success rates for comprehensive efficacy assessment were $73.33 \%$ and $70 \%$ for meropenem and $53.85 \%$ and $61.54 \%$ for tigecycline at EOT visit and upon discharge visit, respectively. Also, no significant treatment differences were observed between two groups.

\section{The secondary outcomes}

30-day all-cause mortality and 60-day all-cause mortality were $10 \%$ to $16.67 \%$ for meropenem group and $11.54 \%$ to $11.54 \%$ for tigecycline group, respectively $(\mathrm{P}>0.05)$. There were no significant differences between the two groups with regard to hospital LOS, ICU LOS (Table 2).

\section{Safety and tolerability}

The most frequently reported AEs were digestive system disorders, including nausea, vomiting, diarrhea and rash and antibacterials-induced Fungal infection.

There were no statistically significant differences 


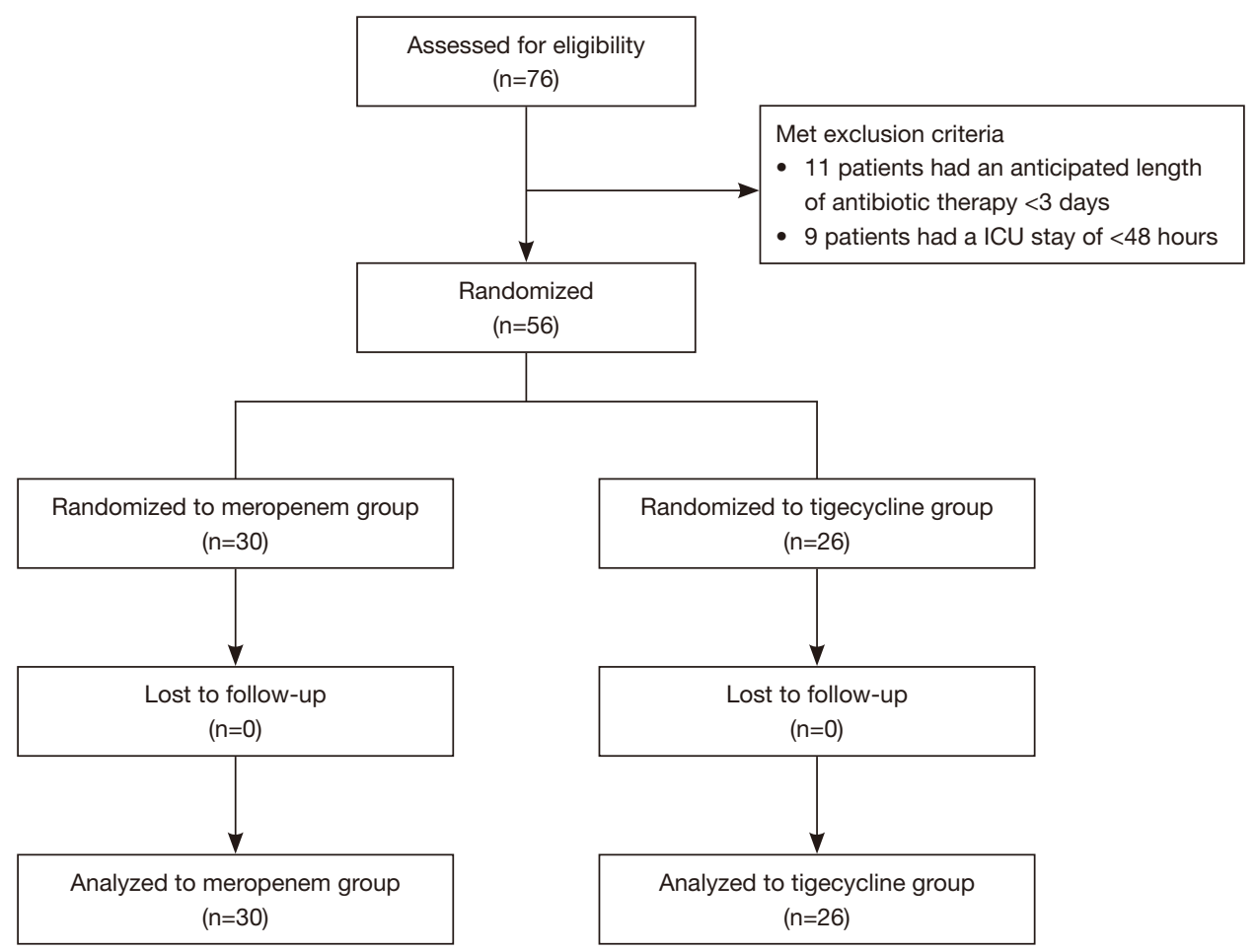

Figure 1 Consolidated standards of reporting trails (CONSORT) flow diagram describing eligibility screening, random allocation, followup and analysis.

Table 1 Demographic and baseline clinical characteristics

\begin{tabular}{|c|c|c|c|}
\hline Characteristics & Meropenem group $(n=30)$ & Tigecycline group $(n=26)$ & $P$ value \\
\hline \multicolumn{4}{|l|}{ Gender, n (\%) } \\
\hline Male & $22(73.33)$ & $21(80.75)$ & \\
\hline Female & $8(26.67)$ & $5(19.25)$ & 0.511 \\
\hline Hypertension & $11(36.67)$ & $11(42.31)$ & 0.666 \\
\hline Diabetes mellitus & $7(23.33)$ & $4(15.38)$ & 0.455 \\
\hline $\mathrm{CHD}$ & $2(6.67)$ & $3(11.54)$ & 0.867 \\
\hline COPD & $1(3.33)$ & $1(3.85)$ & 1.000 \\
\hline \multicolumn{4}{|l|}{ Cancer type, n (\%) } \\
\hline Gastric cancer & $13(43.33)$ & $8(30.77)$ & 0.333 \\
\hline Pancreaticodenal cancer & $4(13.33)$ & $4(15.38)$ & 1.000 \\
\hline Colorectal cancer & $10(33.33)$ & $13(50.00)$ & 0.206 \\
\hline Hepatobiliary neoplasm & $3(10.00)$ & $1(3.85)$ & 0.710 \\
\hline
\end{tabular}

Table 1 (continued) 
Table 1 (continued)

\begin{tabular}{|c|c|c|c|}
\hline Characteristics & Meropenem group $(n=30)$ & Tigecycline group $(n=26)$ & $P$ value \\
\hline Unplanned reoperation, n (\%) & $12(40.00)$ & $12(46.15)$ & 0.643 \\
\hline Serum albumin at admission ICU $(\mathrm{g} / \mathrm{L})$ & $23.15 \pm 5.11$ & $26.03 \pm 3.74$ & 0.128 \\
\hline APACHE II score on admission & $10.67 \pm 3.65$ & $13.12 \pm 5.43$ & 0.05 \\
\hline SOFA score on admission & $5.80 \pm 2.68$ & $5.69 \pm 3.26$ & 0.893 \\
\hline \multicolumn{4}{|l|}{ Types of CIAls, n (\%) } \\
\hline Anastomotic fistula & $17(56.67)$ & $13(50.0)$ & 0.618 \\
\hline Intra-abdominal abscess & $5(16.67)$ & $3(11.54)$ & 0.870 \\
\hline AKI & $9(30.0)$ & $8(30.77)$ & 0.950 \\
\hline Bloodstream infections & $8(26.67)$ & $5(19.23)$ & 0.511 \\
\hline \multicolumn{4}{|l|}{ Multiple organ support in ICU, n (\%) } \\
\hline Vasoactive agents & $21(70.0)$ & $17(65.38)$ & 0.712 \\
\hline Mechanical ventilation & $17(56.67)$ & $18(69.23)$ & 0.333 \\
\hline CVVH & $4(13.33)$ & $5(19.23)$ & 0.815 \\
\hline \multicolumn{4}{|l|}{ Source control, n (\%) } \\
\hline Monomicrobial & $9(30.0)$ & $8(30.77)$ & 0.950 \\
\hline Polymicrobial & $18(60.0)$ & $11(42.31)$ & 0.186 \\
\hline MDR organisms & $8(26.67)$ & $4(15.38)$ & 0.305 \\
\hline De-escalation, n (\%) & & & 0.799 \\
\hline Yes & $9(30.0)$ & 7 (26.92) & \\
\hline No & $21(70.0)$ & $19(73.08)$ & \\
\hline \multicolumn{4}{|l|}{ Initial appropriate antibiotics, n (\%) } \\
\hline Yes & $24(80.0)$ & $17(65.38)$ & 1.000 \\
\hline No & $3(10.0)$ & $2(7.69)$ & \\
\hline Postoperative abdominal bleeding, n (\%) & $8(26.67)$ & $9(34.62)$ & 0.519 \\
\hline Antibiotic duration (days) & $8.37 \pm 3.44$ & $7.19 \pm 3.23$ & 0.196 \\
\hline
\end{tabular}

CHD, coronary artery heart disease; COPD, chronic obstructive pulmonary disease; APACHE II, Acute Physiology and Chronic Health Evaluation; SAPS3, Simplified Acute Physiology Score 3; SOFA, Sequential Organ Failure Assessment; AKI, acute kidney injury; CVVH, continuous veno-venous hemofiltration; MDR, multidrug-resistant. 
Table 2 Comparison of clinical outcomes and adverse events in the two groups

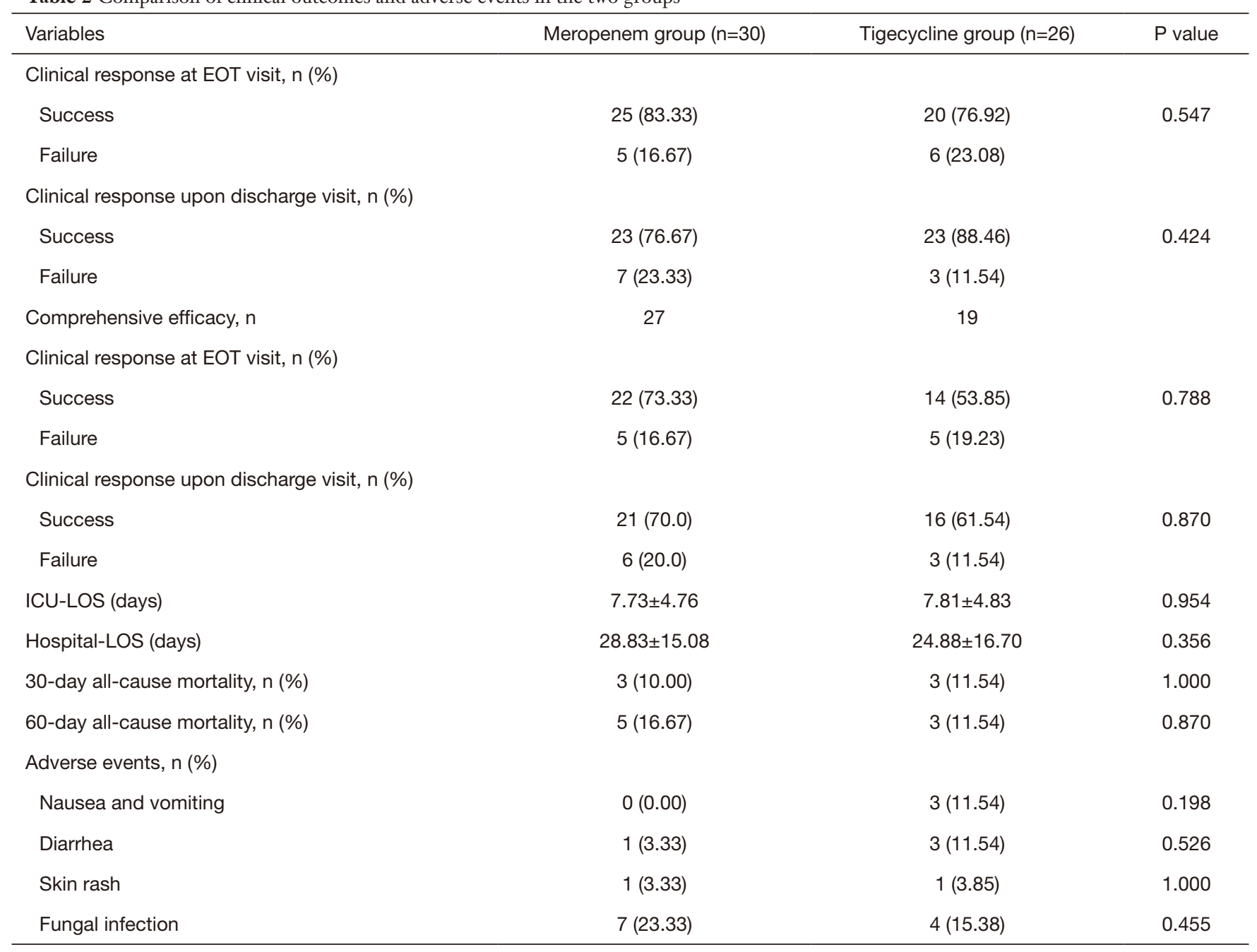

EOT, the end-of-therapy visit; LOS, hospital length of stay.

between treatment groups in the overall incidence of AEs. The overall incidence of AEs in digestive system was 3.33\% for tigecycline vs. $23.0 \%$ for meropenem therapy $(\mathrm{P}>0.05)$. The majority of these AEs were mild to moderate in intensity, patients in trail study could be well tolerated, and no withdrawal occurred (Table 2).

\section{Microbiologic distribution between meropenem and tigecycline groups}

A total of 107 micro-organisms were isolated from samples in two groups, of which 62 isolates were cultured in the meropenem group and 45 pathogens were identified in the tigecycline group (Table 3). Gram-negative organisms constituted $49.53 \%$, Gram-positive bacteria made up
$39.25 \%$, and Candida species comprised $11.21 \%$ of the isolated pathogens. The major pathogens were $E$. coli and Pseudomonas aeruginosa among Gram-negative isolated. The two groups had similar distribution of infecting microorganisms, except for E. coli, Pseudomonas aeruginosa on Gram-positive and Enterococcus species MRSA on Gram-negative and fungi isolates, which were isolated more frequently in meropenem group than tigecycline group. The most commonly isolated pathogens with resistance was Escherichia coli, followed by MRSA and Pseudomonas aeruginosa. Four pathogens comprised 3 strains of Pseudomonas aeruginosa and 1 strains Proteus mirabilis were recovered on culture from 4 patients in tigecycline group, in which 3 patients were cured, one patient failed. 
Table 3 Microbiological distribution between two groups

\begin{tabular}{|c|c|c|c|}
\hline Isolated micro-organisms & Meropenem group $(n=30)$ & Tigecycline group & $P$ value $(n=26)$ \\
\hline Escherichia coli & $11(36.67)$ & 7 (26.92) & 0.436 \\
\hline Pseudomonas aeruginosa & $5(16.67)$ & $3(11.54)$ & 0.870 \\
\hline Klebsiella pneumoniae & $4(13.33)$ & $3(11.54)$ & 1.000 \\
\hline Stenotrophomonas maltophilia & $3[10]$ & $1(3.85)$ & 0.710 \\
\hline Acinetobacter bacteria & $1(3.33)$ & $2(7.69)$ & 0.899 \\
\hline Klebsiella oxytocas & $1(3.33)$ & 2 (7.69) & 0.899 \\
\hline Serratia marcescens & $1(3.33)$ & $1(3.85)$ & 1.000 \\
\hline Enterococcus faecalis & $11(36.67)$ & $10(38.46)$ & 0.890 \\
\hline Enterococcus faecium & $8(26.67)$ & $5(19.23)$ & 0.511 \\
\hline MRSA & $3[10]$ & $1(3.85)$ & 0.710 \\
\hline MRSCON & $1(3.33)$ & $1(3.85)$ & 1.000 \\
\hline Staphylococcus haemolyticus & $1(3.33)$ & $0(0)$ & 1.000 \\
\hline Enterococcus durans & $1(3.33)$ & $0(0)$ & 1.000 \\
\hline Fungi, n (\%) & $7(23.33)$ & $5(19.23)$ & 0.709 \\
\hline Candida albicans & 3 [10] & $4(15.38)$ & 0.839 \\
\hline
\end{tabular}

MRSA, methicillin-resistant Staphylococcus aureus; MRSCON, methicillin-resistant coagulase-negative staphylococci.

\section{Subgroup analysis for clinical outcome in patients with positive isolates of microorganisms}

Microorganisms were isolated from 46 of 56 patients (82.14\%) for whom culture samples were available (Table 4). Clinical success rates stratified by monomicrobial, polymicrobial and MDR infections are founded in Table 4. In this subgroup analysis, the clinical success rates at EOT visit and upon discharge visit had no significant different between the two groups. In particular, regarding MDROs infection, clinical success rates at EOT and upon discharged were $87.5 \%, 75 \%$ for meropenem group and $75 \%, 100 \%$ for tigecycline group $(\mathrm{P}>0.05)$, respectively.

\section{Risk factors associated with 60-day mortality using univariable and multivariable logistic regression analysis}

There was no significant difference in 60-day allcause mortality among patients who were treated with meropenem versus treatment with tigecycline $(16.67 \%$ versus $11.54 \% \mathrm{P}>0.05)$. The results of the univariate analysis showed that serum albumin at admission to ICU, colorectal cancer on oncology type, postoperative abdominal bleeding were associate with increase 60-day all-cause mortality (Table 5).

Multivariate analysis showed that postoperative abdominal bleeding $(\mathrm{P}=0.028$, OR $=14.577,95 \%$ CI: 1.338 
Table 4 Clinical success rates of positive isolates in the two groups

\begin{tabular}{|c|c|c|c|}
\hline Variable & Meropenem group $(n=30)$ & Tigecycline group $(n=26)$ & $P$ value \\
\hline Clinical success at EOT visit, n (\%) & $22(81.48)$ & $14(73.68)$ & 0.788 \\
\hline Clinical success at upon discharge visit, n (\%) & $21(77.78)$ & $16(84.21)$ & 0.870 \\
\hline Monomicrobial infection, $\mathrm{n}(\%)$ & $9(30.0)$ & $8(30.77)$ & \\
\hline Clinical success at upon discharge visit, $\mathrm{n}(\%)$ & $8(88.89)$ & $7(87.5)$ & 1.000 \\
\hline Polymicrobial infection, $\mathrm{n}(\%)$ & $18(60.0)$ & $11(42.31)$ & \\
\hline Clinical success at EOT visit, $n(\%)$ & $15(83.33)$ & $7(63.63)$ & 0.375 \\
\hline Clinical success at upon discharge visit, $\mathrm{n}(\%)$ & $13(72.22)$ & $9(81.82)$ & 0.677 \\
\hline Clinical success at upon discharge visit, $n(\%)$ & $6(75.00)$ & $4[100]$ & 0.515 \\
\hline
\end{tabular}

EOT, the end-of-therapy visit; MDR, multidrug-resistant.

158.828) was the only independent predictors of 60-day allcause mortality (Table 6).

\section{Discussion}

In general, carbapenems have been considered the most active antibiotic against pathogens that cause IAIs. However, the prevalence of carbapenem resistance has increased in certain regions and become a critical issue in hospitals worldwide (19). The sensitive balance between the improvement of empirical antibiotic therapy, which proved to promote better clinical results, and the decrease of needless antimicrobial overuse, related to the increasing development of MDR pathogens, is permanently needed when treating cIAIs (20). So, it is critical to select a suitable antibiotic for the management of cIAIs.

In our previous retrospective study involved 80 postoperative abdominal tumor patients with cIAIs, we found that hospital mortality and ICU mortality were significantly lower in tigecycline group than in meropenem (18). In this study we compared the clinical efficacy between meropenem and tigecycline, and found that tigecycline is efficacious and well-tolerated in the treatment of postoperative abdominal tumors patients with cIAIs. The clinical success rates of tigecycline group was higher than that of meropenem group at upon discharge visit and comprehensive efficacy. However, no significant difference was reached. From the point of this view, both tigecycline and meropenem are feasible in the treatment of postoperative cIAIs of abdominal tumor patients. A Bayesian network meta-analysis of randomized clinical trials showed that on differences in clinical and microbiological outcomes and mortality observed between meropenem and tigecycline (21), which supported our conclusion.

Our data showed that clinical success rate of subgroup of bloodstream infections were $75 \%$ for meropenem and $100 \%$ for tigecycline at upon discharge visit, the difference was not significant. No died case due to BSIs was reported in tigecycline group, which suggested that tigecycline was also very effective against BSIs. A systematic review and meta-analysis showed (22) that clinical cure was significantly higher in the tigecycline regimen, compared with comparator regimen $(\mathrm{P}=0.001)$. Although the overall mortality did not differ between tigecycline and the control groups, subgroup analysis clearly indicated that the mortality was significantly lower in the tigecycline combination group than in the tigecycline monotherapy therapy group. Therefore, a combination therapy with tigecycline may be a fine option for the treatment of BSIs.

In the present study, in patients caused by MDR and polymicrobial infections, clinical success rate for tigecycline was significantly higher than that for meropenem at upon discharge visit, whereas there was no statistical difference. A surveillance trial about tigecycline against MDR Gram- 
Table 5 Univariate analyses of risk factors associated 60-day all-cause mortality

\begin{tabular}{|c|c|c|c|}
\hline Characteristics & Non-survivors group $(n=8)$ & Survivors group $(n=48)$ & P Value \\
\hline Gender, n (\%) & & & 0.220 \\
\hline Male & $8[100]$ & 35 (72.92) & \\
\hline Female & $0(0.00)$ & $13(27.08)$ & \\
\hline Hypertension & $2(25)$ & $22(45.83)$ & 0.615 \\
\hline Diabetes mellitus & $1(12.5)$ & $10(20.83)$ & 0.945 \\
\hline $\mathrm{CHD}$ & $1(12.5)$ & $4(8.33)$ & 0.552 \\
\hline COPD & $0(0.0)$ & $2(4.17)$ & 1.000 \\
\hline Pancreaticodenal cancer & $2(25.0)$ & $6(12.5)$ & 0.697 \\
\hline Colorectal cancer & $0(0.0)$ & $23(47.92)$ & 0.031 \\
\hline Hepatobiliary neoplasm & $1(12.5)$ & $3(6.25)$ & 0.470 \\
\hline Preoperative (chemo-) radiotherapy, n (\%) & $2(25.0)$ & $12(25.0)$ & 1.000 \\
\hline Readmission to ICU, n (\%) & $3(37.5)$ & $10(20.83)$ & 0.561 \\
\hline Unplanned reoperation, n (\%) & $4(50.0)$ & $20(41.67)$ & 0.956 \\
\hline Serum albumin at admission ICU (g/L) & $20.925 \pm 5.791$ & $24.54 \pm 4.21$ & 0.038 \\
\hline APACHE II score on admission & $14.38 \pm 7.41$ & $11.38 \pm 4.03$ & 0.297 \\
\hline CVVH, n (\%) & $3(37.5)$ & $6(12.5)$ & 0.207 \\
\hline Bloodstream infections, n (\%) & $1(12.5)$ & $12(25.0)$ & 0.747 \\
\hline \multicolumn{4}{|l|}{ Source control, n (\%) } \\
\hline Percutaneous drainages & $3(37.5)$ & $6(12.5)$ & 0.207 \\
\hline Surgical procedure & $4(50.0)$ & $14(29.17)$ & 0.448 \\
\hline Abdominal irrigation & $1(12.5)$ & $7(14.58)$ & 1.000 \\
\hline No intervention & $1(12.5)$ & $23(47.92)$ & 0.137 \\
\hline \multicolumn{4}{|l|}{ Type of micro-organisms, n (\%) } \\
\hline Monomicrobial & $1(12.5)$ & $16(33.33)$ & 0.441 \\
\hline Polymicrobial & $6(12.5)$ & $23(47.92)$ & 0.300 \\
\hline MDR organisms & $2(25.0)$ & $10(20.83)$ & 1.000 \\
\hline
\end{tabular}

Table 5 (continued) 
Table 5 (continued)

\begin{tabular}{|c|c|c|c|}
\hline Characteristics & Non-survivors group $(\mathrm{n}=8)$ & Survivors group $(n=48)$ & P Value \\
\hline ICU-LOS & $9.750 \pm 7.21$ & $7.438 \pm 4.23$ & 0.404 \\
\hline Hospital-LOS & $21.875 \pm 11.99$ & $27.854 \pm 16.32$ & 0.327 \\
\hline The presence of Initial appropriate antibiotics, n (\%) & $5(62.5)$ & $36(75.0)$ & 0.160 \\
\hline Postoperative abdominal bleeding, $\mathrm{n}(\%)$ & 7 (87.5) & $10(20.83)$ & 0.001 \\
\hline Categories, n (\%) & & & 0.870 \\
\hline Tigecycline group & $3(37.5)$ & $23(47.92)$ & \\
\hline Meropenem group & $5(62.5)$ & $25(50.08)$ & \\
\hline
\end{tabular}

CHD, coronary artery heart disease; COPD, chronic obstructive pulmonary disease; APACHE II, Acute Physiology and Chronic Health Evaluation; SAPS3, Simplified Acute Physiology Score 3; SOFA, Sequential Organ Failure Assessment; AKI, acute kidney injury; CVVH, continuous veno-venous hemofiltration; MDR, multidrug-resistant.

Table 6 Multivariate analyses of the risk factors associated with 60-day all-cause mortality

\begin{tabular}{lccccc}
\hline Variables & $\beta$ & SE & Wald & OR & $95 \% \mathrm{Cl}$ \\
\hline Serum albumin at admission ICU $(\mathrm{g} / \mathrm{L})$ & -0.057 & 0.112 & 0.266 & 0.944 & $0.759-1.175$ \\
Type cancer: colorectal cancer & -19.271 & 7747.487 & 0.000 & 0.000 & 0.606 \\
Postoperative abdominal bleeding & 2.679 & 1.219 & 4.835 & 14.577 & $1.338-158.828$ \\
\hline
\end{tabular}

ICU, intensive care unit; $\beta$, partial regression coefficient; SE, standard error; OR, odds ratio; Cl, confidence interval.

negative pathogens revealed (23) tigecycline shows the in vitro antimicrobial activity against the majority of Gramnegative bacteria, such as MDR Acinetobacter baumannii, Klebsiella pneumoniae, Escherichia coli, Enterobacter aerogenes, and Enterobacter cloacae. Furthermore, with respect to many Gram-positive and MDR pathogens like Staphylococcus aureus, Enterococci, MRAS, VRE, tigecycline had been proved to retain good vitro activity (24). Another previously published systematic review and metaanalysis demonstrated that tigecycline combination therapy was associated with significantly lower 30-day mortality than does monotherapy (25). Combined our results and literatures, we concluded that tigecycline may be more appropriate than meropenem in the treatment of cIAIs caused by MDR pathogens and polymicrobial infections.

In this study, we found that postoperative abdominal bleeding remained as an independent risk factor of 60-day all-cause mortality in the multivariable analysis. Postoperative hemorrhage following abdominal tumor surgery was a potentially fatal complication, and was associated with high mortality rate of up to $54 \%$ (26). Our study showed that 7 patients in non-survivors group presented postoperative abdominal bleeding, with the mortality rates up to $87.5 \%$. In a study by Martin Loos et al., postoperative abdominal bleeding tended to association with complications like abdominal infection and fistula, whereas fistula and abdominal infections closely related to postoperative bleeding $(27,28)$. Postoperative fistula was more likely to be infected with polymicrobial as well as MDR, including MRSA, Enterococci spp., Enterobacter species, Klebsiella spp., Candida spp., which resulted in significantly higher morbidity and mortality (29). Therefore, for abdominal tumor patients suffering postoperative abdominal bleeding, the empiric use of broad-spectrum antibiotics should be considered as soon as possible to control suspected or documented abdominal infection. On the other hand, transcatheter arterial embolization (TAE) should be considered as the choice of 
management when possible in addition to timely and early surgical interventions (26).

AEs were monitored in this study. Digestive system disorders, antibacterials-induced fungal infection, skin rash were the 3 most frequently reported AEs in both treatment groups. The majority of these AEs were mild to moderate in intensity. The AEs rates were $30 \%$ for meropenem and $42.3 \%$ for tigecycline respectively, however, patients in our study were well tolerated, and no withdrawal occurred. No SAEs were reported.

There were several limitations in our study. Firstly, the design of this study was single center randomized controlled trial, in which fewer patients were enrolled. Moreover, the actual sample size assigned to randomization was less than our calculated sample size because more participants met the exclusion criteria than we predicted. We could not find enough patients eligible for inclusion to the study within a limited period of study. Accordingly, the power of the statistical analysis may have been reduced, which may also be subject to bias. Secondly, the study had not monitored pharmacokinetics and susceptibility data for tigecycline and meropenem. Thirdly, the study was conducted in a tertiary cancer center, which involved only cancer patents. It implied that our results were probably not representative. Lastly, outcome measures assessed antibiotic treatment for CIAIs had not a precise reference standard, and a consensus definition of clinical outcome did not exist and based on subjective measures. So the current outcomes were prone to bias. The standardized outcomes should be developed and implemented, so-called "core outcome sets" (30), that were clinical meaningful would standardize the outcome for cIAIs, and cause better quality evidence production, and enable the clinicians to make better decisions on antimicrobial prescribing of cIAIs.

\section{Conclusions}

In summary, tigecycline was comparable to meropenem. Both drugs were effective and well-tolerated therapy option in treating postoperative cIAIs in cancer patients. Further multi-center, large sample size RCTs are needed to substantiate our findings.

\section{Acknowledgments}

Funding: This project was supported by Clinical Research Fund of Wu Jieping Medical Foundation (No. 320.6750.18033).

\section{Footnote}

Reporting Checklist: The authors have completed the CONSORT reporting checklist. Available at http://dx.doi. org/10.21037/apm-20-907

Data Sharing Statement: Available at http://dx.doi. org/10.21037/apm-20-907

Peer Review File: Available at http://dx.doi.org/10.21037/ apm-20-907

Conflicts of Interest: All authors have completed the ICMJE uniform disclosure form (available at http://dx.doi. org/10.21037/apm-20-907). The authors have no conflicts of interest to declare.

Ethical Statement: The authors are accountable for all aspects of the work in ensuring that questions related to the accuracy or integrity of any part of the work are appropriately investigated and resolved. The study was approved by Independent Ethics Committee of National Cancer Center/Cancer Hospital, Chinese Academy of Medical Sciences and Peking Union Medical College (No. NCC2018W-001), and all patients signed informed consent. The study was conducted in accordance with the Declaration of Helsinki (as revised in 2013).

Open Access Statement: This is an Open Access article distributed in accordance with the Creative Commons Attribution-NonCommercial-NoDerivs 4.0 International License (CC BY-NC-ND 4.0), which permits the noncommercial replication and distribution of the article with the strict proviso that no changes or edits are made and the original work is properly cited (including links to both the formal publication through the relevant DOI and the license). See: https://creativecommons.org/licenses/by-nc-nd/4.0/.

\section{References}

1. Boldingh QJ, de Vries FE, Boermeester MA. Abdominal sepsis. Curr Opin Crit Care 2017;23:159-66.

2. Lin SY, Huang CH, Ko WC, et al. Recent developments in antibiotic agents for the treatment of complicated intra-abdominal infections. Expert Opin Pharmacother 2016;17:339-54.

3. Chen Y, Zhu D, Zhang Y, et al. A multicenter, doubleblind, randomized, comparison study of the efficacy 
and safety of tigecycline to imipenem/cilastatin to treat complicated intra-abdominal infections in hospitalized subjects in China. Ther Clin Risk Manag 2018;14:2327-39.

4. Sartelli M, Chichom-Mefire A, Labricciosa FM, et al. The management of intra-abdominal infections from a global perspective:2017 WSES guidelines for management of intra-abdominal infections. World J Emerg Surg 2017;12:29.

5. Sartelli M, Weber DG, Ruppé E, et al. Antimicrobials: a global alliance for optimizing their rational use in intraabdominal infections (AGORA). World J Emerg surg 2016;11:33.

6. Oshima T, Kodama Y, Takahashi W, et al. Empiric Antibiotic Therapy for Severe Sepsis and Septic Shock. Surg Infect (Larchmt) 2016;17:210-6.

7. De Rosa FG, Corcione S, Perri GD, et al. Re-defining tigecycline therapy. New Microbiol 2015;38:121-36.

8. Schulz KF, Altman DG, Moher D, et al. CONSORT 2010 statement: updated guidelines for reporting parallel group randomised trials. BMJ 2010;340:698-702.

9. Ahmed S, Wilcox MH, Kirby A. Measuring outcomes in complicated intra-abdominal infections. Curr Opin Gastroenterol 2020;36:1-4.

10. National Healthcare Safety Network (NHSN). Patient safety component manual: Centers for Disease Control and Prevention, 2019. Available online: https://www.cdc. gov/nhsn/pdfs/pscmanual/pcsmanual_current.pdf

11. Timsit JF, Ruppé E, Barbier F, et al. Bloodstream infections in critically ill patients: an expert statement. Intensive Care Med 2020;46:266-84.

12. Maharaj R, Terblanche M, Vlachos S, et al. The Utility of ICU Readmission as a Quality Indicator and the Effect of Selection. Crit Care Med 2018;46:749-56.

13. Tellor B, Skrupky LP, Symons W, et al. Inadequate source control and inappropriate antibiotics are key determinants of mortality in patients with intra-abdominal sepsis and associated bacteremia. Surg Infect (Larchmt) 2015;16:785-93.

14. Liu H, Zhou Z. Management Strategy of Intra-Abdominal Infection Caused by Multidrug-Resistant Bacteria. Zhonghua Wei Chang Wai Ke Za Zhi 2018;21:1351-5.

15. Tasu JP, Vesselle G, Herpe G, et al. Postoperative abdominal bleeding. Diagn Interv Imaging 2015;96:823-31.

16. Solomkin JS, Mazuski JE, Bradley JS, et al. Diagnosis and management of complicated intra-Abdominal infection in adults and children: guidelines by the Surgical Infection Society and the Infectious Diseases Society of America. Clin Infect Dis 2010;50:133-64.
17. Food and Drug Administration. Complicated intraabdominal infections: developing drugs for treatment guidance for industry. [Online]. 2018. Available online: https://www.fda.gov/regulatory-information/searchfda-guidance-documents/complicated-intra-abdominalinfections-developing-drugs-treatment.pdf (Accessed 1 January 2020).

18. Wang HJ, Xing XZ, et al. Effects of tigecycline and carbapenems on treatment of complicated intraabdominal infection. Chinese Journal of Nosocomiology 2019;29:2143-53.

19. Martin A, Fahrbach K, Zhao Q, et al. Association Between Carbapenem Resistance and Mortality Among Adult, Hospitalized Patients With Serious Infections Due to Enterobacteriaceae: Results of a Systematic Literature Review and Meta-analysis. Open Forum Infect Dis 2018;5:ofy150.

20. Silva-Nunes J, Cardoso T. Intra-abdominal infections: the role of different classifications on the selection of the best antibiotic treatment. BMC Infect Dis 2019;19:980.

21. Chen L, Liang X, Jiang J, et al. Carbapenems vs tigecycline for the treatment of complicated intraabdominal infections: A Bayesian network meta-analysis of randomized clinical trials. Medicine (Baltimore) 2019;98:e17436.

22. Wang J, Pan Y, Shen J, et al. The efficacy and safety of tigecycline for the treatment of bloodstream infections: a systematic review and meta-analysis. Ann Clin Microbiol Antimicrob 2017;16:24.

23. Giammanco A, Calà C, Fasciana T, et al. Global assessment of the activity of tigecycline against multidrugresistant Gram-negative pathogens between 2004 and 2014 as part of the Tigecycline Evaluation and Surveillance Trial. Msphere 2017;2:e00310-6.

24. Veeraraghavan B, Poojary A, Shankar C, et al. In-vitro activity of tigecycline and comparator agents against common pathogens: Indian experience. J Infect Dev Ctries 2019;13:245-50.

25. Ni W, Han Y, Liu J, et al. Tigecycline treatment for carbapenem-resistant Enterobacteriaceae infections: a systematic review and meta-analysis. Medicine (Baltimore) 2016;95:e3126.

26. Chatani S, Inoue A, Ohta S, et al. Transcatheter arterial embolization for postoperative bleeding following abdominal surgery. Cardiovasc Intervent Radiol 2018;41:1346-55.

27. Yang J, Zhang X, Huang YH, et al. Diagnosis and treatment of abdominal arterial bleeding after radical 
gastrectomy: a retrospective analysis of 1875 consecutive resections for gastric cancer. J Gastrointest Surg 2016;20:510-20.

28. Chen JF, Xu SF, Zhao W, et al. Diagnostic and therapeutic strategies to manage post-pancreaticoduodenectomy hemorrhage. World J Surg 2015;39:509-15.

Cite this article as: Wang HJ, Xing XZ, Qu SN, Huang CL, Zhang H, Wang H, Yang QH, Yuan ZN. A randomized controlled trial comparing the efficacy of tigecycline versus meropenem in the treatment of postoperative complicated intra-abdominal infections. Ann Palliat Med 2021;10(2):1262-1275. doi: 10.21037/apm-20-907
29. Loos M, Strobel O, Legominski M, et al. Postoperative pancreatic fistula: Microbial growth determines outcome. Surgery 2018;164:1185-90.

30. Williamson PR, Altman DG, Bagley H, et al. The COMET Handbook: version 1.0. Trials 2017;18:280. 